<Back to results | 1 of 1

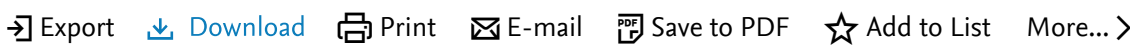

Full Text |View at Publisher|

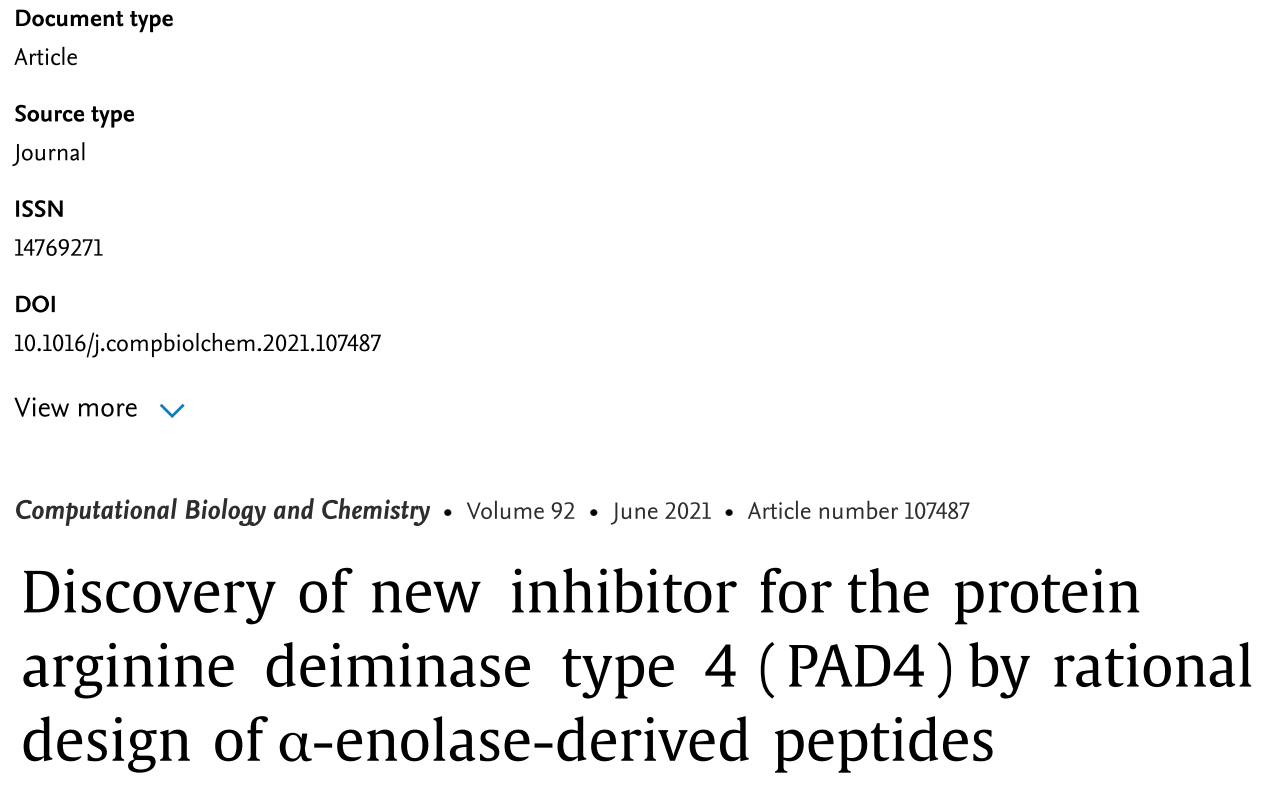

Computational Biology and Chemistry • Volume 92 • June 2021 • Article number 107487

\section{Discovery of new inhibitor for the protein arginine deiminase type 4 (PAD4) by rational design of $\alpha$-enolase-derived peptides}

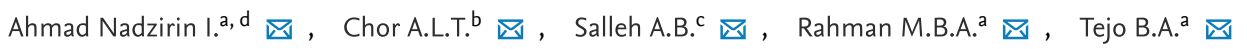
国 Save all to author list

a Department of Chemistry, Faculty of Science, Universiti Putra Malaysia, UPM, Serdang, 43400, Malaysia

${ }^{b}$ Department of Cell and Molecular Biology, Faculty of Biotechnology and Biomolecular Sciences, Universiti Putra Malaysia, UPM, Serdang, 43400, Malaysia

c Department of Biochemistry, Faculty of Biotechnology and Biomolecular Sciences, Universiti Putra Malaysia, UPM, Serdang, 43400, Malaysia

${ }^{d}$ Department of Biomedical Science, Faculty of Allied Health Science, International Islamic University Malaysia, Kuantan, 25200, Pahang, Malaysia

\section{3}

Views count ?

View all metrics >
Cited by 0 documents

Inform me when this document is cited in Scopus:

Set citation alert >

Related documents

Structure-based design of peptide inhibitors for protein arginine deiminase type iv (PAD4)

Ying, T.C. , Ibrahim, Z. , Abd Rahman, M.B.

(2018) Encyclopedia of Bioinformatics and

Computational Biology: $A B C$ of Bioinformatics

A fluopol-ABPP HTS assay to identify PAD inhibitors

Knuckley, B. , Jones, J.E. , Bachovchin, D.A. (2010) Chemical Communications

Haloacetamidine-based inactivators of protein arginine deiminase 4 (PAD4): Evidence that general acid catalysis promotes efficient inactivation

Knuckley, B. , Causey, C.P. , Pellechia, P.J.

(2010) ChemBioChem

View all related documents based on references

Find more related documents in Scopus based on:

Authors > Keywords >

\title{
Abstract
}

Author keywords

Reaxys Chemistry database information

Indexed keywords

SciVal Topics

Chemicals and CAS Registry Numbers

Metrics

Funding details 
Rheumatoid arthritis (RA) is an inflammatory autoimmune disease affecting about $0.24 \%$ of the world population. Protein arginine deiminase type 4 ( PAD4) is believed to be responsible for the occurrence of RA by catalyzing citrullination of proteins. The citrullinated proteins act as autoantigens by stimulating an immune response. Citrullinated $\alpha$-enolase has been identified as one of the autoantigens for RA. Hence, $\alpha$-enolase serves as a suitable template for design of potential peptide inhibitors against PAD4 . The binding affinity of $\alpha$-enolase-derived peptides and PAD4 was virtually determined using PatchDock and HADDOCK docking programs. Synthesis of the designed peptides was performed using a solid phase peptide synthesis method. The inhibitory potential of each peptide was determined experimentally by PAD4 inhibition assay and $\mathrm{IC}_{50}$ measurement. PAD4 assay data show that the N-P2 peptide is the most favourable substrate among all peptides . Further modification of N-P2 by changing the Arg residue to canavanine [P2 (Cav)] rendered it an inhibitor against PAD4 by reducing the PAD4 activity to $35 \%$ with $\mathrm{IC}_{50} 1.39 \mathrm{mM}$. We conclude that P2 (Cav) is a potential inhibitor against PAD4 and can serve as a starting point for the development of even more potent inhibitors. (C) 2021 Elsevier Ltd

Author keywords

Drug design; PAD4; Peptide inhibitor; Rheumatoid arthritis

Reaxys Chemistry database information (i)

Substances

View all substances $(8)$
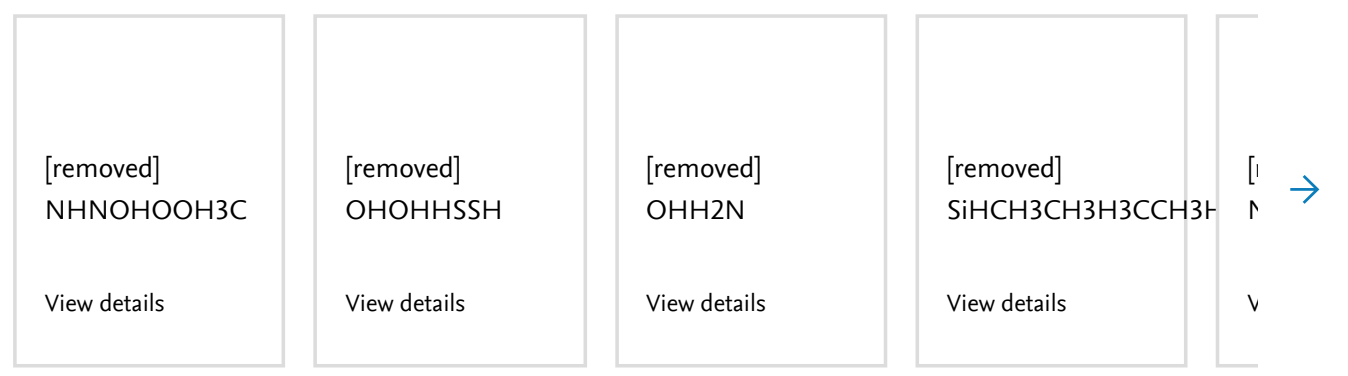

Powered by Reaxys

Indexed keywords

SciVal Topics (i)

Chemicals and CAS Registry Numbers

Metrics

Funding details

References (40)

View in search results format $>$

All

Export 合Print 四-mail 哭 Save to PDF Create bibliography

1 Andrusier, N., Nussinov, R., Wolfson, H.J.

FireDock: Fast interaction refinement in molecular docking

(2007) Proteins: Structure, Function and Genetics, 69 (1), pp. 139-159. Cited

438 times.

doi: $10.1002 /$ prot.21495

View at Publisher

2 Badillo-Soto, M.A., Rodríguez-Rodríguez, M., Pérez-Pérez, M.E.

Potential protein targets of the peptidylarginine deiminase 2 and

peptidylarginine deiminase 4 enzymes in rheumatoid synovial tissue and its possible meaning

(2016) Eur. J. Rheumatol., 3 (2), pp. 44-49. Cited 13 times. 
3 Blachère, N.E., Parveen, S., Frank, M.O., Dill, B.D., Molina, H., Orange, D.E. High-Titer Rheumatoid Arthritis Antibodies Preferentially Bind Fibrinogen Citrullinated by Peptidylarginine Deiminase 4 (Open Access)

(2017) Arthritis and Rheumatology, 69 (5), pp. 986-995. Cited 24 times. http://onlinelibrary.wiley.com/journal/10.1002/(ISSN) 1529-0131 doi: 10.1002/art.40035

View at Publisher

4 Bradford, M.M.

A rapid and sensitive method for the quantitation of microgram quantities of protein utilizing the principle of protein-dye binding

(1976) Analytical Biochemistry, 72 (1-2), pp. 248-254. Cited 205559 times. doi: 10.1016/0003-2697(76)90527-3

View at Publisher

5 Burmester, G.R., Pope, J.E.

Novel treatment strategies in rheumatoid arthritis

(2017) The Lancet, 389 (10086), pp. 2338-2348. Cited 289 times. http://www.journals.elsevier.com/the-lancet/

doi: 10.1016/50140-6736(17)31491-5

View at Publisher

6 Cross, M., Smith, E., Hoy, D., Carmona, L., Wolfe, F., Vos, T., Williams, B., (...), March, L.

The global burden of rheumatoid arthritis: Estimates from the Global Burden of Disease 2010 study

(2014) Annals of the Rheumatic Diseases, 73 (7), pp. 1316-1322. Cited 549

times.

http://ard.bmj.com/content/73/7/1316.full.pdf

doi: 10.1136/annrheumdis-2013-204627

View at Publisher

7 Crowson, C.S., Matteson, E.L., Myasoedova, E., Michet, C.J., Ernste,

F.C., Warrington, K.J., Davis III, J.M., (...), Gabriel, S.E.

The lifetime risk of adult-onset rheumatoid arthritis and other inflammatory autoimmune rheumatic diseases (Open Access)

(2011) Arthritis and Rheumatism, 63 (3), pp. 633-639. Cited 271 times. doi: 10.1002/art.30155

View at Publisher

8 Curran, A.M., Naik, P., Giles, J.T., Darrah, E.

PAD enzymes in rheumatoid arthritis: pathogenic effectors and autoimmune targets

(2020) Nature Reviews Rheumatology, 16 (6), pp. 301-315. Cited 15 times. http://www.nature.com/nrrheum/archive/index.html doi: $10.1038 / 541584-020-0409-1$

View at Publisher

9 Curtis, J.R., Wallenstein, G., Takiya, L., Gruben, D., Chen, C., Shan, Y., Blachley, T., (...), Kremer, J.

Patterns of methotrexate use and discontinuation in a U.S. Rheumatoid arthritis registry [abstract]

(2017) Arthritis Rheumatol., 69. Cited 3 times. 
10 Gellman, S.H., Woolfson, D.N.

Mini-proteins trp the light fantastic

(2002) Nature Structural Biology, 9 (6), pp. 408-410. Cited 56 times.

doi: $10.1038 / \mathrm{nsb} 0602-408$

View at Publisher

11 Harris, M.L., Darrah, E., Lam, G.K., Bartlett, S.J., Giles, J.T., Grant, A.V., Gao, P., (...), Rosen, A.

Association of autoimmunity to peptidyl arginine deiminase type 4 with genotype and disease severity in rheumatoid arthritis (Open Access)

(2008) Arthritis and Rheumatism, 58 (7), pp. 1958-1967. Cited 93 times. doi: 10.1002/art.23596

View at Publisher

12 Hua, J., Huang, W.

Peptidylarginine deiminase $4-104 \mathrm{C} / \mathrm{T}$ polymorphism and risk of rheumatoid arthritis: A pooled analysis based on different populations (Open Access)

(2018) PLoS ONE, 13 (3), art. no. e0193674. Cited 7 times.

http://journals.plos.org/plosone/article/file?

id=10.1371/journal.pone.0193674\&type=printable

doi: $10.1371 /$ journal.pone.0193674

View at Publisher

13 Hunter, T.M., Boytsov, N.N., Zhang, X., Schroeder, K., Michaud, K., Araujo, A.B.

Prevalence of rheumatoid arthritis in the United States adult population in healthcare claims databases, 2004-2014

(2017) Rheumatology International, 37 (9), pp. 1551-1557. Cited 148 times. link.springer.de/link/service/journals/00296/index.htm doi: 10.1007/s00296-017-3726-1

View at Publisher

14 Kearney, P.L., Bhatia, M., Jones, N.G., Yuan, L., Glascock, M.C., Catchings, K.L., Yamada, M., (...), Thompson, P.R.

Kinetic characterization of protein arginine deiminase 4: A transcriptional corepressor implicated in the onset and progression of rheumatoid arthritis

(2005) Biochemistry, 44 (31), pp. 10570-10582. Cited 145 times. doi: $10.1021 /$ bi050292m

View at Publisher

15 Kinloch, A., Tatzer, V., Wait, R., Peston, D., Lundberg, K., Donatien, P., Moyes, D., (...), Venables, P.J.

Identification of citrullinated alpha-enolase as a candidate autoantigen in rheumatoid arthritis.

(2005) Arthritis research \& therapy, 7 (6), pp. R1421-1429. Cited 278 times.

View at Publisher

16 Knuckley, B., Bhatia, M., Thompson, P.R.

Protein arginine deiminase 4: Evidence for a reverse protonation mechanism (Open Access)

(2007) Biochemistry, 46 (22), pp. 6578-6587. Cited 68 times. doi: $10.102 \mathrm{l} / \mathrm{bi700095}$

View at Publisher 
17 Knuckley, B., Luo, Y., Thompson, P.R.

Profiling Protein Arginine Deiminase 4 (PAD4): A novel screen to identify PAD4 inhibitors (Open Access)

(2008) Bioorganic and Medicinal Chemistry, 16 (2), pp. 739-745. Cited 61

times.

doi: 10.1016/j.bmc.2007.10.021

View at Publisher

18 Kurowska, W., Przygodzka, M., Jakubaszek, M., Kwiatkowska, B., Maslinski, W. Interleukin-15 as a biomarker candidate of rheumatoid arthritis development (Open Access)

(2020) Journal of Clinical Medicine, 9 (5), art. no. 1555. Cited 4 times. https://www.mdpi.com/2077-0383/9/5/1555/pdf doi: $10.3390 / \mathrm{jcm} 9051555$

View at Publisher

19 Lee, Y.H., Bae, S.-C.

Association between susceptibility to rheumatoid arthritis and PADI4 polymorphisms: A meta-analysis

(2016) Clinical Rheumatology, 35 (4), pp. 961-971. Cited 18 times. link.springer.de/link/service/journals/10067/index.htm doi: 10.1007/s10067-015-3098-4

View at Publisher

20 Li, L., Li, Z., Chen, D., Lu, X., Feng, X., Wright, E.C., Solberg, N.O., (...), Zhang, L.

Inactivation of microbial arginine deiminases by L-canavanine (2008) Journal of the American Chemical Society, 130 (6), pp. 19181931. Cited 34 times. doi: $10.1021 / j a 0760877$

View at Publisher

21 Lu, C., Xu, K., Guo, H., Peng, K., Yang, Z., Hao, Y.Q., Xu, P.

The relationship of PADI4_94 polymorphisms with the morbidity of rheumatoid arthritis in Caucasian and Asian populations: a meta-analysis and system review

(2018) Clinical Rheumatology, 37 (2), pp. 289-296. Cited 7 times. link.springer.de/link/service/journals/10067/index.htm doi: 10.1007/s10067-017-3964-3

View at Publisher

22 Luo, Y., Arita, K., Bhatia, M., Knuckley, B., Lee, Y.-H., Stallcup, M.R., Sato, M., (...), Thompson, P.R.

Inhibitors and inactivators of protein arginine deiminase 4: Functional and structural characterization (Open Access)

(2006) Biochemistry, 45 (39), pp. 11727-11736. Cited 192 times. doi: 10.1021/bi061180d

View at Publisher 
23 Luo, Y., Knuckley, B., Lee, Y.-H., Stallcup, M.R., Thompson, P.R.

A fluoroacetamidine-based inactivator of protein arginine deiminase 4: Design, synthesis, and in vitro and in vivo evaluation (Open Access)

(2006) Journal of the American Chemical Society, 128 (4), pp. 1092-

1093. Cited 107 times.

doi: $10.1021 / j a 0576233$

View at Publisher

24 Mashiach, E., Schneidman-Duhovny, D., Andrusier, N., Nussinov, R., Wolfson, H.J.

FireDock: a web server for fast interaction refinement in molecular docking. (Open Access)

(2008) Nucleic acids research, 36 (Web Server issue), pp. W229-232. Cited 410 times.

doi: $10.1093 / \mathrm{nar} / \mathrm{gkn1} 186$

View at Publisher

25 Maupetit, J., Derreumaux, P., Tufféry, P.

A fast method for large-scale de novo peptide and miniprotein structure prediction

(2010) Journal of Computational Chemistry, 31 (4), pp. 726-738. Cited 155 times.

http://www3.interscience.wiley.com/cgi-bin/fulltext/122474624/PDFSTART doi: $10.1002 /$ jcc.21365

View at Publisher

26 Miller, M.-C., Manning, H.B., Jain, A., Troeberg, L., Dudhia, J., Essex D., Sandison, A., (...), Itoh, Y.

Membrane type 1 matrix metalloproteinase is a crucial promoter of synovial invasion in human rheumatoid arthritis (Open Access)

(2009) Arthritis and Rheumatism, 60 (3), pp. 686-697. Cited 88 times. http://www3.interscience.wiley.com/cgi-bin/fulltext/122220648/PDFSTART doi: $10.1002 /$ art.24331

View at Publisher

27 Pap, T., Shigeyama, Y., Kuchen, S., Fernihough, J.K., Simmen, B., Gay, R.E., Billingham, M., (...), Gay, S.

Differential expression pattern of membrane-type matrix metalloproteinases in rheumatoid arthritis (Open Access)

(2000) Arthritis and Rheumatism, 43 (6), pp. 1226-1232. Cited 119 times. doi: 10.1002/1529-0131(200006)43:6<1226::AID-ANR5>3.0.CO;2-4

View at Publisher

28 Rodríguez, S.B., Stitt, B.L., Ash, D.E.

Cysteine 351 is an essential nucleophile in catalysis by Porphyromonas gingivalis peptidylarginine deiminase (Open Access)

(2010) Archives of Biochemistry and Biophysics, 504 (2), pp. 190-196. Cited 6 times.

doi: 10.1016/j.abb.2010.09.008

View at Publisher 
29 Rückert, R., Brandt, K., Bulanova, E., Mirghomizadeh, F., Paus, R., BulfonePaus, $S$.

Dendritic cell-derived IL-15 controls the induction of CD8 T cell immune reponses

(2003) European Journal of Immunology, 33 (12), pp. 3493-3503. Cited 77

times.

doi: $10.1002 /$ eji.200324545

View at Publisher

30 Schneidman-Duhovny, D., Inbar, Y., Nussinov, R., Wolfson, H.J.

PatchDock and SymmDock: Servers for rigid and symmetric docking (Open Access)

(2005) Nucleic Acids Research, 33 (SUPPL. 2), pp. W363-W367. Cited 1734

times.

doi: $10.1093 /$ nar/gki481

View at Publisher

31 Shen, Y., Maupetit, J., Derreumaux, P., Tufféry, P.

Improved PEP-FOLD approach for peptide and miniprotein structure prediction

(2014) Journal of Chemical Theory and Computation, 10 (10), pp. 4745-

4758. Cited 299 times.

http://pubs.acs.org/journal/jctcce

doi: $10.1021 /$ ct500592m

View at Publisher

32 Smolen, J.S., Aletaha, D., Machold, K.P.

Therapeutic strategies in early rheumatoid arthritis

(2005) Best Practice and Research: Clinical Rheumatology, 19 (1), pp. 163-

177. Cited 75 times.

doi: 10.1016/j.berh.2004.08.009

View at Publisher

33 Stefano, A., Tolusso, B., Petricca, L., Ferraccioli, G., Gremese, E.

Chapter 46 - Rheumatoid Arthritis,Editor(s): Carlo Perricone, Yehuda

Shoenfeld, Mosaic of Autoimmunity

(2019), pp. 501-526.

Academic Press

34 Suzuki, A., Kochi, Y., Shoda, H., Seri, Y., Fujio, K., Sawada, T., Yamada, R., (...), Yamamoto, K.

Decreased severity of experimental autoimmune arthritis in peptidylarginine deiminase type 4 knockout mice (Open Access)

(2016) BMC Musculoskeletal Disorders, 17 (1), art. no. 205. Cited 32 times. http://www.biomedcentral.com/bmcmusculoskeletdisord/ doi: 10.1186/s12891-016-1055-2

View at Publisher

35 Takahara, H., Okamoto, H., Sugawara, K.

Affinity chromatography of peptidylarginine deiminase from rabbit skeletal muscle on a column of soybean trypsin inhibitor (kunitz)-sepharose

(1986) Journal of Biochemistry, 99 (5), pp. 1417-1424. Cited 36 times. doi: 10.1093/oxfordjournals.jbchem.al35611

View at Publisher 
36 Teo, C.Y., Shave, S., Chor, A.L., Salleh, A.B., Rahman, M.B., Walkinshaw, M.D., Tejo, B.A.

Discovery of a new class of inhibitors for the protein arginine deiminase type 4 (PAD4) by structure-based virtual screening. (Open Access)

(2012) BMC bioinformatics, 13 Suppl 17, p. S4. Cited 17 times. doi: 10.1186/1471-2105-13-s17-s4

View at Publisher

37 Teo, C.Y., Tejo, B.A., Leow, A.T.C., Salleh, A.B., Abdul Rahman, M.B.

Novel furan-containing peptide-based inhibitors of protein arginine deiminase type IV (PAD4) (Open Access)

(2017) Chemical Biology and Drug Design, 90 (6), pp. 1134-1146. Cited 5

times.

http://www.wiley.com/bw/journal.asp?ref=1747-0277

doi: $10.1111 /$ cbdd.13033

View at Publisher

38 Thévenet, P., Shen, Y., Maupetit, J., Guyon, F., Derreumaux, P., Tufféry, P. PEP-FOLD: An updated de novo structure prediction server for both linear and disulfide bonded cyclic peptides

(Open Access)

(2012) Nucleic Acids Research, 40 (W1), pp. W288-W293. Cited 339 times. doi: $10.1093 / \mathrm{nar} / \mathrm{gks} 419$

View at Publisher

39 van Venrooij, W.J., Pruijn, G.J.M.

An important step towards completing the rheumatoid arthritis cycle (Open Access)

(2008) Arthritis Research and Therapy, 10 (5), art. no. 117. Cited 28 times. doi: 10.1186/ar2504

View at Publisher

40 Van Zundert, G.C.P., Rodrigues, J.P.G.L.M., Trellet, M., Schmitz, C., Kastritis, P.L., Karaca, E., Melquiond, A.S.J., (...), Bonvin, A.M.J.J.

The HADDOCK2.2 Web Server: User-Friendly Integrative Modeling of Biomolecular Complexes (Open Access)

(2016) Journal of Molecular Biology, 428 (4), pp. 720-725. Cited 840 times. https://www.journals.elsevier.com/journal-of-molecular-biology. doi: 10.1016/j.jmb.2015.09.014

View at Publisher

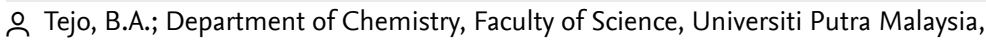
UPM, Serdang, Malaysia; email:bimo.tejo@upm.edu.my

(C) Copyright 2021 Elsevier B.V., All rights reserved.

\section{About Scopus}

What is Scopus

Content coverage

Scopus blog

Scopus API

Privacy matters

\section{Language}

日本語に切り替える

切换到简体中文

切換到繁體中文

Русский язык

\section{Customer Service}

Help

Contact us 
Copyright (C) Elsevier B.V ז. All rights reserved. Scopus ${ }^{\circledR}$ is a registered trademark of Elsevier B.V.

We use cookies to help provide and enhance our service and tailor content. By continuing, you agree to the

use of cookies. 